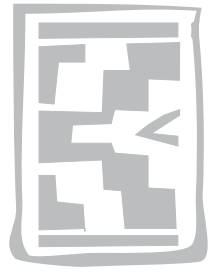

\title{
Comparison of the survival on ice of thawed Theileria parva sporozoites of different stocks cryoprotected by glycerol or sucrose
}

\author{
V. MBAO ${ }^{1}$, D. BERKVENS ${ }^{2}$, P. DORNY ${ }^{2,3}$, P. VAN DEN BOSSCHE ${ }^{2,4}$ and T. MARCOTTY2*
}

\begin{abstract}
MBAO, V., BERKVENS, D., DORNY, P., VAN DEN BOSSCHE, P. \& MARCOTTY, T. 2007. Comparison of the survival on ice of thawed Theileria parva sporozoites of different stocks cryoprotected by glycerol or sucrose. Onderstepoort Journal of Veterinary Research, 74:9-15

Stabilates of Theileria parva sporozoites are mostly delivered in liquid nitrogen tanks to the East Coast fever immunization points. Using an in vitro titration model, we assessed the loss of infectivity of several stabilates when they are stored in ice baths for up to $24 \mathrm{~h}$. Comparisons, with respect to rates of loss of infectivity, were made between T. parva stocks (Chitongo and Katete), cryoprotectants (sucrose and glycerol) and method of assessment (in vivo and in vitro techniques). Chitongo and Katete stabilates showed similar loss dynamics. The losses were 1-4\% (depending on parasite stock) and $3 \%$ per hour of storage for glycerol and sucrose stabilates respectively, and the loss rates were not significantly different. The results suggest that Chitongo stabilates and sucrose cryoprotected suspensions can be delivered on ice as is done for Katete. A graphical relationship of in vitro effective dose at $50 \%$ infectivity $\left(\mathrm{ED}_{50}\right)$ and in vivo protection rate was made. The relationship showed a $35 \%$ loss of protection for a relatively low corresponding increase of $E D_{50}$ from 0.006 to 0.007 tick equivalent.
\end{abstract}

Keywords: Cold-chain, immunization, in vitro, sporozoites, Theileria parva, Zambia

\section{INTRODUCTION}

East Coast fever, an often fatal disease of cattle in the eastern, central and southern parts of Africa, is

* Author to whom correspondence is to be directed. E-mail: tmarcotty@itg.be

1 Department of Veterinary and Livestock Development, Ministry of Agriculture and Cooperatives, P.O. Box 670050, Mazabuka, Zambia. Present address: Department of Animal Health, Institute of Tropical Medicine, Nationalestraat 155, B-2000 Antwerp, Belgium

2 Department of Animal Health, Institute of Tropical Medicine, Nationalestraat 155, B-2000 Antwerp, Belgium

3 Department of Parasitology, Faculty of Veterinary Medicine, Ghent University, Salisburylaan 133, B9820 Merelbeke, Belgium

4 Department of Veterinary Tropical Diseases, Faculty of Veterinary Science, University of Pretoria, Private Bag X04, Onderstepoort, 0110 South Africa

Accepted for publication 15 September 2006-Editor caused by Theileria parva, an obligate intracellular protozoan parasite. It is mostly transmitted by the three-host ixodid ticks Rhipicephalus appendiculatus and Rhipicephalus zambeziensis. The disease is a major constraint to livestock development in the affected regions (Young, Groocock \& Kariuki 1988). Control methods include immunization by the Infection-and-Treatment Method (I\&T) (Radley, Brown, Burridge, Cunningham, Kirimi, Purnell \& Young 1975) in which doses of $T$. parva cryopreserved stabilates (Cunningham, Brown, Burridge \& Purnell 1973) are inoculated simultaneously with a long acting tetracycline. The method is widely applied in the field (Uilenberg 1999; Marcotty, Billiouw, Chaka, Berkvens, Losson \& Brandt 2001; Fandamu, Thys, Duchateau \& Berkvens 2006).

Currently, stabilates are stored in liquid nitrogen. The maintenance of the cold chain up to the farm level is complicated which makes the method less 
appropriate for rural circumstances and is expensive for livestock keepers. This problem was partly alleviated in the Eastern Province of Zambia by storing the stabilate on ice for a few hours between thawing and inoculation. This method allows the distribution of the service using bicycles instead of motorcars. Studies conducted in this region using a glycerated $T$. parva Katete stock stabilate (Geysen, Bishop, Skilton, Dolan \& Morzaria 1999) showed that after $6 \mathrm{~h}$ of storage on ice stabilates could still protect $90 \%$ of the immunized animals (Marcotty et al. 2001). In the southern province of Zambia, the I\&T immunization against ECF makes use of the milder Chitongo strain (Geysen et al. 1999). There is no information on how long stabilates of this $T$. parva strain can be stored on ice without appreciable loss in their potency.

In this study, the loss of infectivity of stabilates due to storage on ice was assessed by in vitro titration (Marcotty, Speybroeck, Berkvens, Chaka, Besa, Madder, Dolan, Losson \& Brandt 2004). The performance of the Chitongo stock was compared with that of the Katete stock with the aim of determining the suitability of the deferred immunization technique using the Chitongo stock.

Various studies indicated that sucrose is a promising $T$. parva stabilate cryoprotectant. It is cheap and more user friendly than glycerol. Ongoing work seems to indicate that sucrose stabilates have higher infectivity, and therefore better infectivity, on recovery from cryopreservation compared to glycerol counterparts (Mbao, unpublished data 2005). Since it is not known whether stabilates cryoprotected by sucrose show similar survival when stored on ice as glycerol-cryoprotected stabilates, the infectivities during ice storage of sucrose and glycerol Katete stabilates were compared.

A considerable amount of work on in vitro assessment of $T$. parva stabilates has been done (Wilkie, Kirvar \& Brown 2002; Marcotty et al. 2004; Mbao, Speybroeck, Berkvens, Dolan, Dorny, Madder, Mulumba, Duchateau, Brandt \& Marcotty 2005). This has shown the potential of the technique as an economical and ethical way of assessing stabilate infectivity for determining immunizing doses, comparison of stocks and evaluating effects of various processes during stabilate production and cryopreservation. However, a relationship between in vitro and in vivo assays to predict actual immunization potential of stabilates is yet to be made. In the present work, we evaluated this link by establishing a graphical relationship between effective doses that give $50 \%$ infectivity $\left(E_{50}\right)$ (in vitro) to proportions of animals successfully immunized (in vivo). When a stabilate is stored on ice, its effective dose for respective levels of infectivity increases with time. These doses can easily be assessed in vitro and compared to the proportions of animals successfully immunized with similar stabilates stored in similar conditions.

\section{MATERIALS AND METHODS}

\section{Stabilate preparations}

\section{Animals}

Two Friesian heifers kept in a tick-proof stall were inoculated with $1 \mathrm{~m} \ell$ of $T$. parva Katete subcutaneously below the right parotidian lymph nodes. They were checked daily for rectal temperature and once the parotidian nodes were palpably swollen, biopsies were aspirated and smears prepared for calculating percentages of schizont-infected lymphoblasts. Thin jugular blood smears were made to check for parasitaemia. Nymphal Rhipicephalus appendiculatus ticks were applied on Day 10 post inoculation. The moulted ticks (adults) were pre-fed for 4 days on New Zealand white rabbits kept at the Institute of Tropical Medicine animal quarters. These ticks were used to produce stabilates $\mathrm{K} 2 \mathrm{~g}$ and $\mathrm{K} 2 \mathrm{~s}$ as described below. Another Friesian heifer was used in a similar way to infect a batch of ticks with $T$. parva Chitongo for the production of stabilate C1g.

\section{Stabilates used}

One Chitongo and three Katete stabilates were used. Katete stabilates were all produced from batches of adult $R$. appendiculatus ticks, infected as nymphs with the same T. parva seed stabilate. The first glycerated Katete stabilate $(\mathrm{K} 1 \mathrm{~g})$ that was used in this study had been produced and tested in vivo by Marcotty et al. (2001). The two other Katete stabilates, $\mathrm{K} 2 \mathrm{~g}$ and $\mathrm{K} 2 \mathrm{~s}$, were produced for the present work from a single batch of infected ticks but were cryoprotected using $7.5 \%(\mathrm{w} / \mathrm{v})$ glycerol and $0.3 \mathrm{M}(10 \%$ $\mathrm{w} / \mathrm{v})$ sucrose, respectively. The Chitongo stabilate (C1g) was cryoprotected with $7.5 \%$ glycerol.

\section{Production}

For all stabilate productions, infected nymphs were allowed to moult in an incubator at $22^{\circ} \mathrm{C}$ and $85 \%$ relative humidity. Eight to 12 weeks after engorgement, the resulting adult ticks were fed on rabbits for 4 days to induce sporogony of the parasite. Harvested ticks were manually ground in a mortar using a pestle for 15 min in Minimum Essential Medium supplemented with Bovine Serum Albumin (MEM/BSA) 
(Purnell, Brown, Cunningham, Burridge, Kirimi \& Ledger 1973) at a concentration of 20 ticks per $1 \mathrm{~m} \ell$. For glycerol stabilates, an equal amount of chilled MEM/BSA with glycerol at $15 \%(\mathrm{w} / \mathrm{v})$ was added drop-wise (OIE 2005). For the sucrose stabilate, an equal amount of $0.6 \mathrm{M}$ sucrose/MEM/BSA solution was added to give final concentration of $0.3 \mathrm{M}$. The $0.6 \mathrm{M}$ sucrose medium had been prepared by dissolving $30.8 \mathrm{~g}$ of sucrose grains (Sigma \#S1888) in $150 \mathrm{~m} \ell$ of MEM/BSA solution. The extracts were stirred continuously in an ice bath. The K2g and K2s stabilates were prepared in a single session and from a single batch of ticks, the only difference being that, at the stage where the cryoprotectant was added, half the extract was mixed with glycerol and the other with sucrose as described above. Some portions of the stabilates were further diluted to a final concentration of one tick per $1 \mathrm{~m} \ell$. All suspensions were aliquoted into $1.5 \mathrm{~m} \ell$ Nalgene ${ }^{\circledR}$ cryogenic vials $(1 \mathrm{~m} \ell$ per vial). Vials were then placed in polystyrene boxes and transferred to $\mathrm{a}-80^{\circ} \mathrm{C}$ freezer for $24 \mathrm{~h}$ before being plunged into liquid nitrogen for storage.

\section{Ice bath storage}

Storage on ice meant keeping stabilate vials in a polystyrene box filled with water and pieces of melting ice (about $3^{\circ} \mathrm{C}$ ). Groups of six vials of each stabilate were thawed at $37^{\circ} \mathrm{C}$ for $5 \mathrm{~min}$ and stored on ice for different periods. The $\mathrm{K} 1 \mathrm{~g}$ stabilate was stored on ice for 3,8 or $24 \mathrm{~h}$. The $\mathrm{C} 1 \mathrm{~g}$ stabilate storage times were $1,3,6,12$ or $24 \mathrm{~h}$. Finally, the thawed K2g and K2s stabilates were kept on ice for 6,12 or $24 \mathrm{~h}$ before the titration. Stabilate for in vivo titration had been stored on ice for $8,12,16,24$ or $32 \mathrm{~h}$ as reported by Marcotty et al. (2001).

\section{In vitro titrations}

Peripheral blood mononuclear cells (PBMC) were isolated from one Friesian heifer throughout the study. The protocol for in vitro titration of T. parva tick-derived stabilates was described by Marcotty et al. (2004) and modified by Mbao et al. (2005).

The K2g and K2s stabilates stored on ice, including a control group (thawed at titration), were transferred to separate falcon tubes and centrifuged. The supernatants, being arbitrarily allocated to separate rows in a 96-well microtitration plate (12 rows by eight columns), were then serially diluted eight times (two-fold dilution). All stabilate groups were titrated in parallel in three sessions. A session was taken as a titration at a given time, sharing a batch of PBMC, culture media and stabilate diluents, thereby forming a cluster for the purpose of statistical analyses (Marcotty et al. 2004). For controls, stabilate freshly thawed from liquid nitrogen storage was used. The $\mathrm{C} 1 \mathrm{~g}$ and $\mathrm{K} 1 \mathrm{~g}$ stabilates were titrated in single sessions. The stabilate was diluted 12 times ( 1.5 fold dilution). Table 1 presents the various sessions and microtitration plate setup.

After 10 days of incubation, $100 \mu \ell$ of each microtitration well were transferred to a separate slide as a cytospin smear. To avoid cross contaminations between various stabilates, each row (corresponding to a different stabilate) was assigned to a particular cyto-centrifuge block and sampling was done from the lowest to the highest stabilate concentration.

Experimental animals were maintained and treated humanely according to the guidelines laid down by the Ethics Commission of the Institute of Tropical Medicine of Antwerp, Belgium (DG003-MM-K-Rip). Details of animals used for production of $\mathrm{K} 1 \mathrm{~g}$ by Marcotty et al. (2001) are fully described in the given reference.

\section{In vivo titrations}

The in vivo titrations data used to compare with in vitro was obtained from Marcotty et al. (2001). The relationship between storage on ice and proportions of successfully immunized animals are illustrated in Fig. 1.

TABLE 1 Sessions and number of microtitration plate wells read for stabilates stored on ice

\begin{tabular}{l|c|c|c|c|}
\hline & $\mathrm{K} 1 \mathrm{~g}$ & $\mathrm{~K} 2 \mathrm{~g}$ & $\mathrm{~K} 2 \mathrm{~s}$ & $\mathrm{C} 1 \mathrm{~g}$ \\
\cline { 2 - 5 } Number of sessions & 1 & $3^{*}$ & 3 & 1 \\
Plates/session & 2 & $4^{*}$ & 4 & 2 \\
Wells read & 188 & 569 & 575 & 120 \\
\hline$\quad$ Same sessions and plates as for K2s & \\
$\mathrm{K}=$ Katete & \\
$\mathrm{C}=$ Chitongo & \\
$\mathrm{g}=$ glycerol &
\end{tabular}




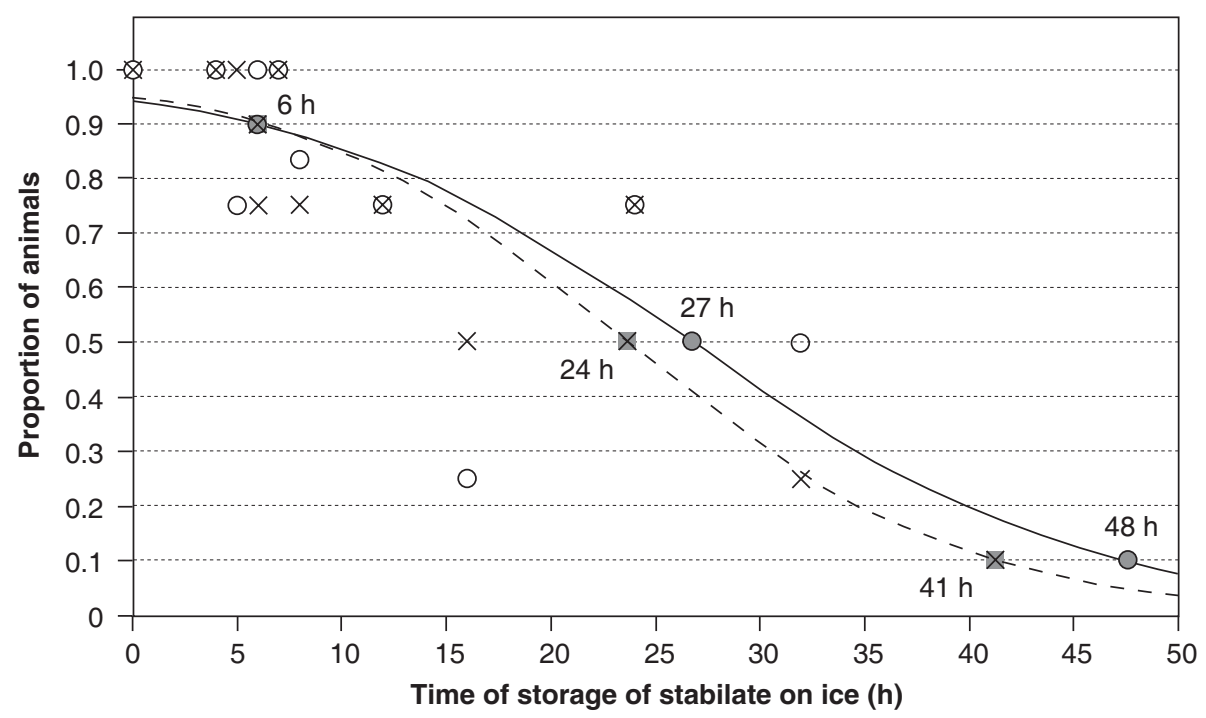

\begin{tabular}{|cl|}
\hline$\times$ & Seroconversion (observed) \\
$\bigcirc$ & Resistance to lethal challenge (observed) \\
-- & Seroconversion (best fit): Logit $(\mathrm{y})=3.0-0.12{ }^{*} \mathrm{x}$ \\
- & Resistance to challenge (best fit): Logit $(\mathrm{y})=2.8-0.1{ }^{*} \mathrm{x}$ \\
$\times$ & T0.1, T0.5 and T0.9 on seroconversion \\
$\mathrm{O}$ & T0.1, T0.5 and T0.9 on resistance to lethal challenge
\end{tabular}

FIG. 1 Effect of storing Theileria parva stabilates on ice on the ability to induce immunity in cattle (Marcotty et al. 2001)

\section{Statistical analysis}

Infectivity losses of Katete and Chitongo (K1g and $C 1 \mathrm{~g}$ ) in function of time of storage on ice

Data were analysed by logistic regression in Stata ${ }^{\circledR}$ (Stata Corporation, Texas). The proportion of positive wells was the response variable and explanatory variables were the dose (natural log of tick equivalents [In t.e.]) and storage time on ice. Storage time was considered both as a discrete variable and, in a simplified model, as a continuous variable. The two models were compared by means of a likelihood ratio test. The level of significance was set at $5 \%$.

Estimation of residual infectivity was conducted by using ratios of effective doses [EDcontrol/EDx] where $\mathrm{x}=$ time of storage on ice. This was calculated using a non-linear combination of estimators which also calculates the ratios' respective confidence intervals (Mbao et al. 2005).

Infectivity losses of glycerol and sucrose stabilates (K2g and K2s) in function of time of storage on ice

The comparison of the infectivity of stabilates cryoprotected with glycerol or sucrose was conducted by a logistic regression using the GLLAMM command (Generalised Linear Latent and Mixed Models) in Stata ${ }^{\circledR}$, with vial and session as random effects. The explanatory variables were stabilate cryoprotectant (sucrose or glycerol), stabilate dose (In t.e.) and time of storage on ice. The response variable was the proportion of positive wells. Interactions between time and stabilate as well as dose and stabilate were tested. Initially, all variables except the dose were entered as discrete variables. This was the saturated model. The non-significant interactions were dropped and the analysis redone with time as continuous variable. This was the simpler model. When the two models were not statistically different $(P>0.05)$, the simpler model was adopted.

Comparison of the viability estimations in in vivo and in vitro experiments (K1g in vivo and in vitro)

Results from the in vivo evaluation of the $\mathrm{K} 1 \mathrm{~g}$ stabilate were obtained from previous experimental work (Marcotty et al. 2001). The best fit curve on observed proportions of protected animals was calculated in a logistic model.

Taking the time of storage as a common axis for the in vivo and in vitro infectivity loss evaluations, the in vitro $\mathrm{ED}_{50}$ estimates were plotted against predicted proportions of protected animals (in vivo) that had been inoculated with $\mathrm{K} 1 \mathrm{~g}$. 


\section{RESULTS}

For all stabilates, the time of storage on ice was taken as a continuous variable as the likelihood ratio tests comparing these models to the models using the time as a discrete variable were not significant $(P>0.05)$.

\section{Infectivity losses of Katete and Chitongo (K1g and $\mathrm{C} 1 \mathrm{~g}$ ) in function of time of storage on ice}

The two stabilates were kept in separate models as they were tested separately. For K1g, the residual infectivity after each hour of storage on ice was estimated to be 0.99 of the infectivity in the preceding hour $(95 \% \mathrm{Cl}$ : $0.96-1.02)$. The effect of storage time was not significant whether time was a discrete variable $(P=0.99, P=0.24$ and $P=0.55$ for times 3,8 and $24 \mathrm{~h}$, respectively) or continuous variable $(P=0.45)$. The residual infectivity of $C 1 \mathrm{~g}$ after storage on ice was 0.96 of the infectivity in the preceding hour (95\% Cl: 0.93-1.00). Similarly, storage time was not significant for discrete time $(P=1, P=$ $1, P=0.17$ and $P=0.17$ for times $3,6,12$ and $24 \mathrm{~h}$, respectively) or continuous time $(P=0.07)$ (Fig. 2).

\section{Infectivity losses of glycerol and sucrose stabilates (K2g and K2s) in function of time of storage on ice}

Since the stabilates were tested in parallel, infectivity losses of the two stabilates were analysed in one

A
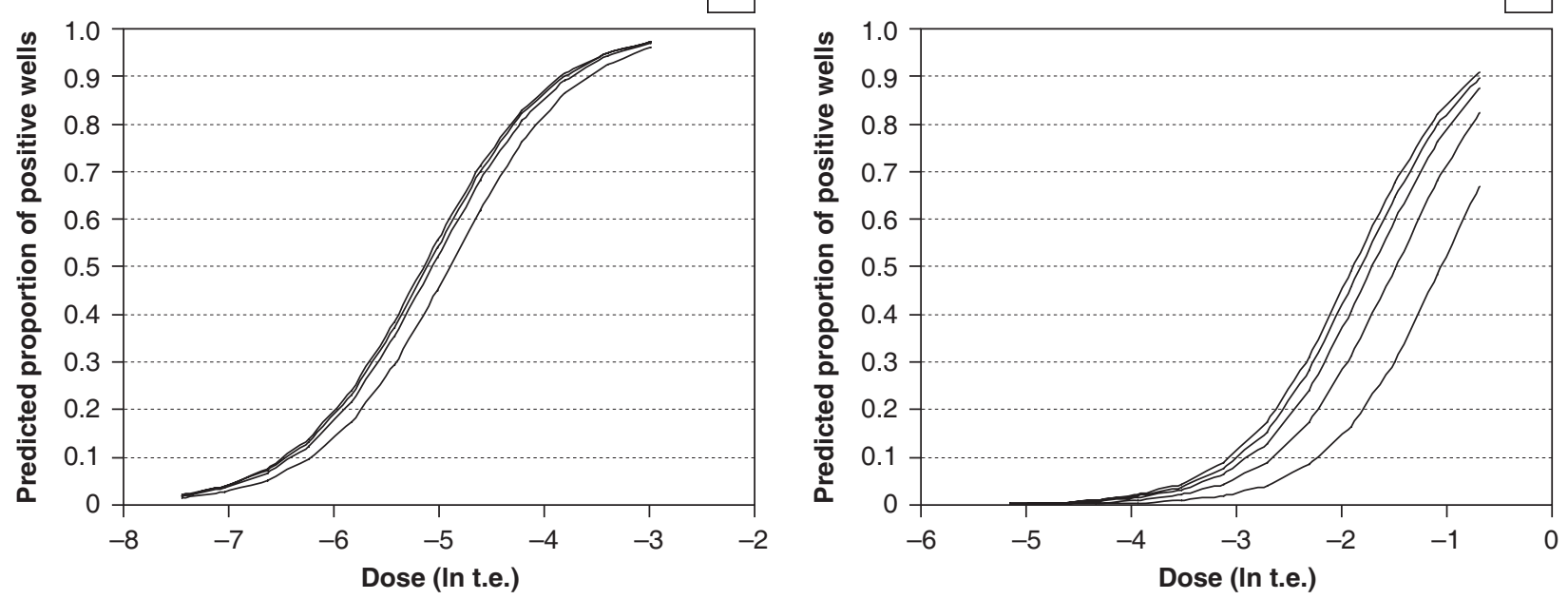

FIG. 2 (A) Titration curves of $\mathrm{K} 1 \mathrm{~g}$ (Katete) stored on ice for $0,3,8$ and $24 \mathrm{~h}$ (from left to right). [In t.e.] is the natural log of the stabilate dose expressed in tick equivalents; and (B) C1g (Chitongo) stored on ice for 1, 3, 6, 12, $24 \mathrm{~h}$ (from left to right)

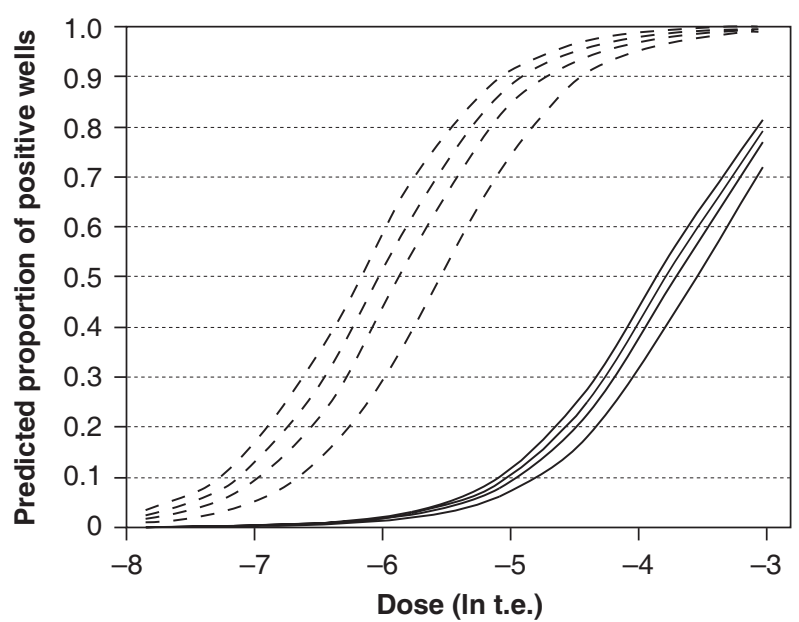

FIG. 3 Titration curves of Katete-K2g (-) and K2s (-----) stabilates stored on ice for 0 (control), 6,12 and $24 \mathrm{~h}$ (from left to right). (In t.e.) is the natural log of the stabilate dose expressed in tick equivalents

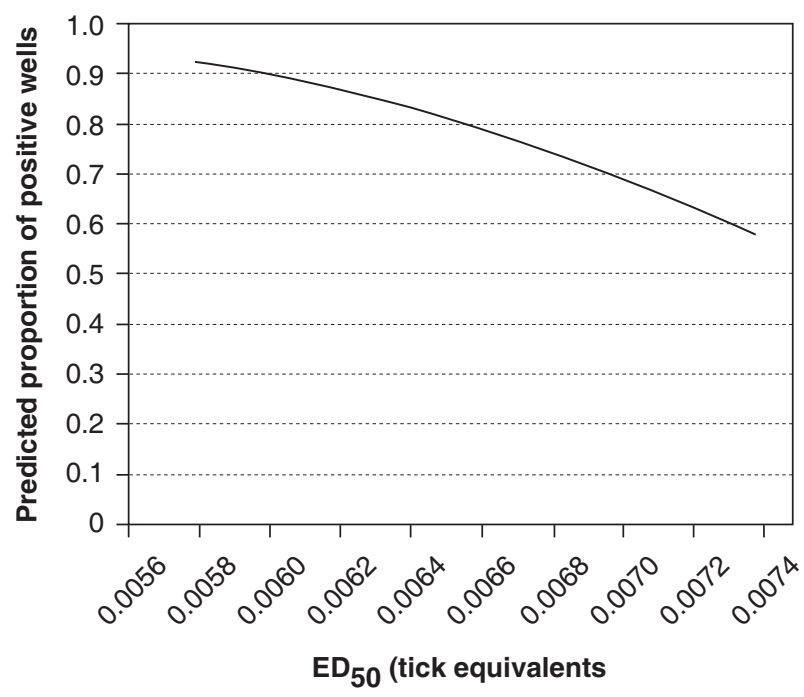

FIG. 4 Correlation between proportions of protected animals against in vitro $\mathrm{ED}_{50}$ 
model. The $\mathrm{K} 2 \mathrm{~g}$ had a residual infectivity of 0.99 of that in the preceding hour of storage. The effect of storage time was not significant $(P=0.35)$ for the studied time periods. The K2s had 0.97 residual infectivity per hour of storage. Here, effect of storage time was significant $(P=0.04)$. The interaction between stabilate and storage time was not significant $(P=0.45)$. Base infectivity, i.e. infectivity after production and cryo-storage (before storage on ice) for sucrose was 10 times higher that of glycerol $(95 \%$ Cl: 6.2-16.7) (Fig. 3).

\section{Comparison of the viability estimations in in vivo and in vitro experiments (K1g in vivo and in vitro)}

The graphical representation shows that an increase of $E_{50}$ from 0.006 to 0.007 tick equivalents (in vitro) results in the protection proportion dropping from $92 \%$ to $57 \%$ (in vivo) (Fig. 4).

\section{DISCUSSION}

Results showed that the loss of infectivity after shortterm storage on ice of Katete and Chitongo strain T. parva stabilates with glycerol or sucrose as cryoprotectant was minimal. This observation is in line with the one of Musisi, Quiroga, Njuguna, Kamwendo \& Chamambala (1996) who found that animals were protected with a trivalent stabilate stored on ice for $15 \mathrm{~h}$. Similar results were obtained by Marcotty et al. (2001) who observed successful immunization in $90 \%$ of cattle inoculated with stabilates stored on ice for up to $6 \mathrm{~h}$. Except for the sucrose-stabilate (K2s), the effect of storage on ice for up to $24 \mathrm{~h}$ was not statistically significant. It is assumed that an effect of storage time on infectivity (reduced infectivity) will be observed for longer storage periods than investigated in this work. However, it was observed that keeping stabilate on ice for longer periods resulted in contamination of the cultures on several occasions. This was most likely due to fungal proliferation (from stabilate tick material) at this temperature.

The observed dynamics of infectivity loss for the Katete and Chitongo strains were similar. This indicates that the deferred immunization technique that has been applied in the Eastern Province of Zambia since 1996 could be used in the Southern Province where the Chitongo stock is used. This would greatly simplify the delivery of stabilate to the remote areas and make delivery much cheaper. Regarding the two different strains used here, the infectivity loss dynamics observed may, therefore, be true for other
T. parva stocks in other regions of Africa. However, it may be necessary to similarly test such stocks to confirm this assumption.

Since the interaction between stabilate and time was not significant when comparing Katete glycerol and sucrose stabilates, it is assumed that the stabilate cryoprotected with sucrose did not lose infectivity faster or slower than the stabilate cryprotected with glycerol. In terms of base infectivity (infectivity at time 0), sucrose stabilate appeared to have higher titres despite having been prepared from the same batch of ticks and during the same production session. This was also seen in comparisons of other glycerol/sucrose stabilates prepared with a similar protocol (Mbao, unpublished data 2005) and may be due to lower toxicity of sucrose for sporozoites. However, the observed difference in titre may also be due to an effect on the host cells, for instance, glycerol may be more toxic to lymphocytes. It would be necessary to conduct a more direct comparison (without the use of lymphocytes) of the effects of these two cryoprotectants for a good conclusion, e.g. by comparing the proportion of live sporozoites on recovery from cryopreservation using reverse transcription-polymerase chain reaction. Sucrose therefore remains a potentially cheaper and better candidate for field stabilates.

A loss of protection in vivo by $35 \%(92-57 \%)$ in going from in vitro $\mathrm{ED}_{50}$ of 0.006 to 0.007 seems to be a drastic loss. In terms of stabilate dilutions, this would be similar to diluting a stabilate by a factor of 1.2 times. Practically, this dilution is too small to account for the corresponding large loss in protection. Cunningham, Brown, Burridge, Musoke Purnell, Radley \& Sempebwa (1974) observed a decrease in protection of only about $10 \%$ on diluting stabilate from $1 / 150$ to $1 / 450$ (3 times dilution). Therefore, it is assumed that the observed anomaly is rather due to the small number of animals that had been used in the in vivo trial (about four animals per storage period). The in vivo trial also lacked repetitions. In the assumption that in vitro result are more accurate, these two factors could have resulted in an underestimation of residual infectivity of the stabilate. On the other hand, the in vitro model assumes that sporozoites are live or dead i.e. infective or not. Some sporozoites might, however, be weakened by storage on ice but remain infective in vitro. The same sporozoites may no longer be infective in vivo because of more hostile conditions like non-specific immune reactions. This would result in an overestimation of residual infectivity if we assume the in vivo result to be more accurate. In short, this lack of 
agreement between in vivo and in vitro observations could be explained by either the lack of accuracy of the in vivo trial or, more likely, by the different conditions to which weakened sporozoites are exposed in the two techniques. Such biases and limitations should be considered carefully when using the in vitro model.

Notwithstanding the considerations outlined in effecting a relationship between the techniques, the in vitro technique remains a valuable alternative to in vivo testing as it remains more ethical and cheaper. Further, in vitro titration/evaluation has the advantage that sessions are easily repeated to offset random variation seen in in vivo titrations. Ideally, parallel in vitro and in vivo trials using the same thawed stabilate and a larger number of animals should be set up, preferably with several repetitions to confirm the relation between in vivo and in vitro T. parva infections.

In conclusion, sucrose protected stabilates lose infectivity at the same rate as glycerol protected ones. Chitongo and Katete strains have similar infectivity losses when stored on ice. Chitongo stabilates used in the Southern Province of Zambia can therefore be delivered to the immunization points in this way (up to $6 \mathrm{~h}$ ). This would reduce the costs and complications associated with stabilate delivery in liquid nitrogen thus making the I \& T immunization option available and affordable to more cattle keepers. Further, this finding could be valid for other strains of $T$. parva in other regions affected by East Coast fever.

\section{ACKNOWLEDGEMENTS}

This work was funded by a Belgian Technical Cooperation PhD scholarship. The authors thank the laboratory staff of the Institute for Tropical Medicine, Antwerp, where the work was done. We are particularly grateful to Anke van Hul for the help in the preparation and reading of microscopic slides.

\section{REFERENCES}

CUNNINGHAM, M.P., BROWN, C.G.D., BURRIDGE, M.J. \& PURNELL, R.E. 1973. Cryopreservation of infective particles of Theileria parva. International Journal for Parasitology, 3: 583-587.
CUNNINGHAM, M.P., BROWN, C.G.D., BURRIDGE, M.J., MUSOKE, A.J., PURNELL, R.E., RADLEY, D.E. \& SEMPEBWA, C. 1974. East Coast fever: Titration in cattle of suspensions of Theileria parva derived from ticks. British Veterinary Journal, 130:336-345.

FANDAMU, P., THYS, E., DUCHATEAU, L. \& BERKVENS, D. 2006. Perception of cattle farmers on East Coast fever immunizations in Southern Zambia. Tropical Animal Health and Production, 38:9-16.

GEYSEN, D., BISHOP, R., SKILTON, R., DOLAN, T.T. \& MORZARIA, S. 1999. Molecular epidemiology of Theileria parva in the field. Tropical Medicine and International Health, 4:A21-A27.

MARCOTTY, T., BILLIOUW, M., CHAKA, G., BERKVENS, D., LOSSON, B. \& BRANDT, J. 2001. Immunization against East Coast fever by the infection and treatment method: evaluation of the use of ice baths for field delivery and appraisal of an acid formulation of long-acting tetracycline. Veterinary Parasitology, 99:175-187.

MARCOTTY, T., SPEYBROECK, N., BERKVENS, D., CHAKA, G., BESA, R.K., MADDER, M., DOLAN, T.T., LOSSON, B. \& BRANDT, J. 2004. In vitro titration of Theileria parva tick derived stabilates. Parasitology, 128:131-137.

MBAO, V., SPEYBROECK, N., BERKVENS, D., DOLAN T., DORNY, P., MADDER, M., MULUMBA, M., DUCHATEAU, L., BRANDT, J. \& MARCOTTY, T. 2005. Comparison of manual and homogenizer methods for preparation of tick-derived stabilates of Theileria parva: equivalence testing using an in vitro titration model. Parasitology, 131:45-49.

MUSISI, F.L., QUIROGA, J.C., NJUGUNA, L.M., KAMWENDO, S.P. \& CHAMAMBALA, K.E. 1996. Infectivity of Theileria parva trivalent stabilate after thawing and maintenance at $4^{\circ} \mathrm{C}$ for up to 15 hours. International Journal for Parasitology, 26:175-179.

OIE 2004. Manual of diagnostic tests and vaccines for terrestrial animals, $5^{\text {th }}$ ed. www.oie.int/fr/normes/mmanual/a_0062.htm (Consulted 20/02/06).

PURNELL, R.E., BROWN, C.G.D., CUNNINGHAM, M.P., BURRIDGE, M.J., KIRIMI, I.M. \& LEDGER, M.A. 1973. East Coast fever: correlation between the morphology and infectivity of Theileria parva developing in its tick vector. Parasitology, 66: 539-544.

RADLEY, D.E., BROWN, C.G.D., BURRIDGE, M.J., CUNNINGHAM, M.P., KIRIMI, I.M., PURNELL, R.E. \& YOUNG, A.S. 1975. East Coast fever: 1. Chemoprophylactic immunization of cattle against Theileria parva (Muguga) and five theilerial strains. Veterinary Parasitology, 1:35-41.

UILENBERG, G. 1999. Immunization against diseases caused by Theileria parva: a review. Tropical Medicine and International Health, 4:A12-A20.

WILKIE, G.M., KIRVAR, E. \& BROWN, C.G.D. 2002. Validation of an in vitro method to determine infectivity of cryopreserved sporozoites in stabilates of Theileria spp. Veterinary Parasitology, 104:199-209.

YOUNG, A.S., GROOCOCK, C.M. \& KARIUKI, D.P. 1988. Integrated control of ticks and tick-borne diseases of cattle in Africa. Parasitology, 96:403-432. 